\title{
Ultrastructure of abnormal membrane inclusions in nuclei of human myocardial cells ${ }^{1}$
}

\author{
HOGNE ENGEDAL, HELGE JENSEN, AND THV. SELMER S ÆTERSDAL \\ From the Cellular Cardiology Research Group, Division of Cardiac Surgery, Bergen University \\ Hospital; and Institute of Anatomy, University of Bergen, Bergen, Norway
}

The ultrastructure of the nuclear envelope was studied in cardiac muscle cells of 12 patients undergoing cardiopulmonary bypass surgery. Two main types of membrane inclusions, globular and tubular, were observed in the nuclei. The globular type was found in 6 patients. The globular inclusions were about $0.5 \mu$ to $1.5 \mu$ in diameter and lined by two unit membranes equal in structure to that of the nuclear envelope. The lining was probably always in continuity with the nuclear membranes. Such inclusions contained a granular matrix in which no cytoplasmic organelles could be seen, with the exception of a limited number of ribosomes. The tubular type of nuclear inclusion was observed in the cardiac tissue of 3 patients. These tubules ran in straight or slightly bent segments of about $0.2 \mu$ to $1.6 \mu$ length, interrupted by bendings at obtuse or right angles. The diameter of their lumina measured about $300 \dot{A}$ to $800 \dot{A}$. Such tubules were nearly always found in areas of the nucleoplasm rich in heterochromatin. Nuclear pore-like structures were occasionally found in the tubular wall. The tubular wall consisted of a single unit membrane shown to be in continuity with the inner membrane of the nuclear envelope. Since these nuclear aberrances have been observed at all of the stages examined, the possibility is considered that they may represent a specific nuclear response to the process of cardiac hypertrophy.

Additional information concerning the mechanism of cardiac enlargement may be gained by studying the ultrastructure of the nuclei of hypertrophied myocardial cells. In a recent publication, Ferrans et al. (1975) showed the presence of abnormal membrane inclusions in nuclei of human myocardial cells. Similar findings have been reported in cell nuclei of a variety of cells (Bernhard and Granboulan, 1963; Clyman, 1963; Terzakis, 1965; Babai et al., 1969). In an ultrastructural study of the hypertrophied human myocardium (Jensen et al., 1976; Saetersdal et al., 1976) we have observed intranuclear membrane structures similar in general appearance to those reported by Ferrans et al. (1975). Our findings, however, vary to a certain extent from those presented in the latter paper. Furthermore, the biopsies of the present study, being taken at three different stages during open heart surgery, allow for a comparison between the presence of nucleic aberrations and the state of

${ }^{1}$ This work was supported by grants from the Norwegian Council on Cardiovascular Diseases and from the Norwegian Research Council for Science and the Humanities.

Received for publication 12 July 1976 ischaemia of the heart wall during cardiopulmonary bypass.

\section{Subjects and methods}

The present observations were made in a group of 12 patients undergoing cardiopulmonary bypass surgery because of congenital septal defect and congenital or acquired valvular disease. In 8 patients examinations were performed on three biopsies from each patient, i.e. at the start and end of aortic crossclamping and after 20 minutes of reperfusion. In 4 patients biopsy was only taken at the start of cardiopulmonary bypass. Biopsies were obtained from the left ventricular wall by the Vim Silverman technique (Rake et al., 1969) using a Tru-Cut biopsy needle (Travenol Laboratories, Inc., Deerfield, Ill. 60015, USA). The anoxic period varied between 30 and 78 minutes during which the heart was protected by local cardiac hypothermia $\left(15^{\circ} \mathrm{C}\right)$.

Specimens were fixed for 3 hours in an ice-cold solution of Karnovsky's fixative (1965) to which 5 per cent sucrose was added. The tissue was washed overnight in a cacodylate-buffered 1 per 
cent sucrose solution before being treated with 1 per cent cacodylate-buffered $\mathrm{OsO}_{4}$ solution for $1 \frac{1}{2}$ hours. Then, after a wash in the same buffer and a wash in distilled water, the tissue was stained en bloc for $1 \frac{1}{2}$ hours in a 2 per cent uranyl acetate solution before being dehydrated through an

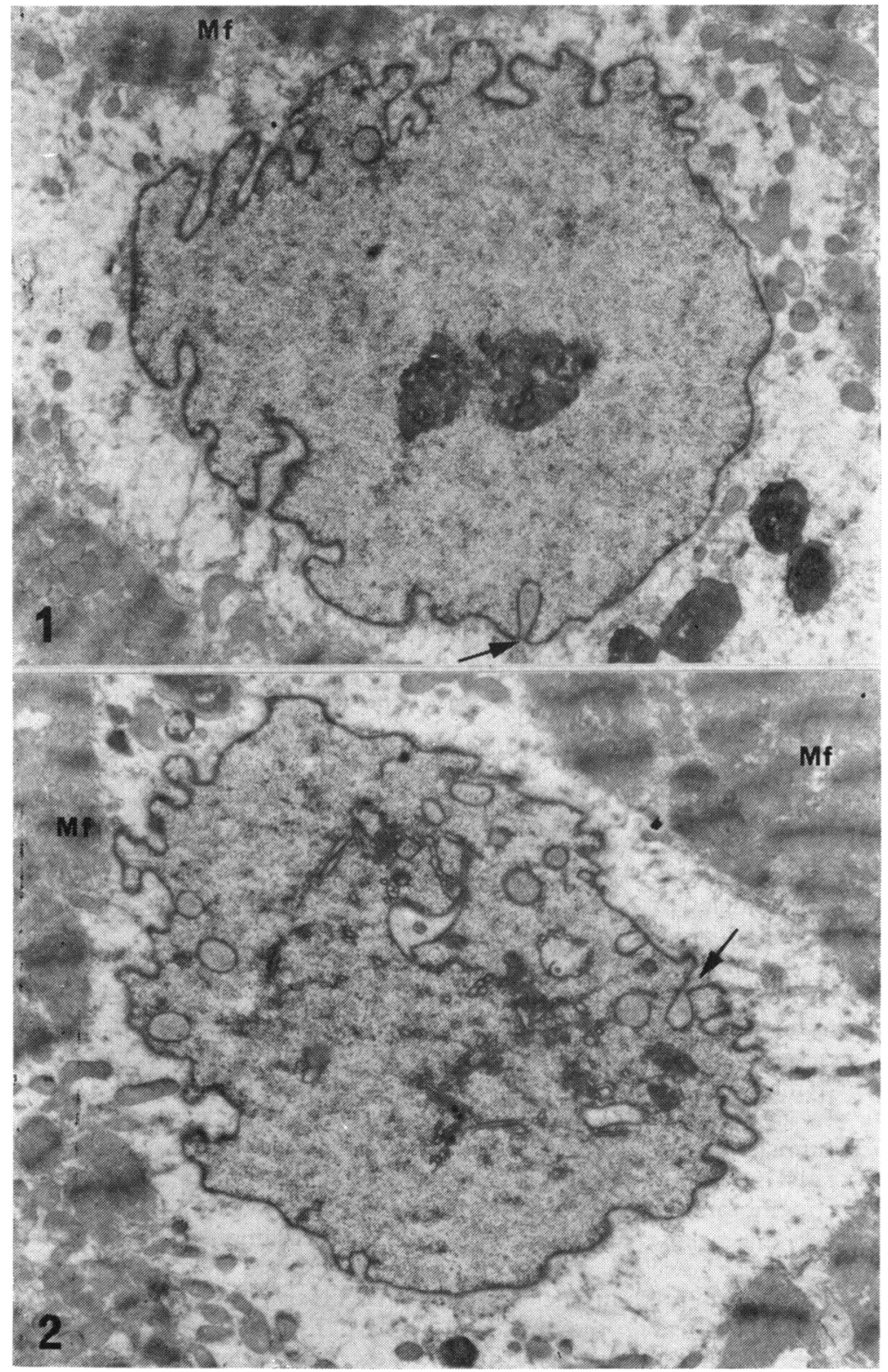

Fig. 1 Myocardial cell nucleus of a 52-year-old patient with aortic valvular stenosis. The lateral surfaces of the nucleus show serrated contraction folds. Two pseudoinclusions are present, one at upper left and another at arrow. The lumen of the latter can be seen to be in continuity with perinuclear area. $M f=$ Myofilaments. $(\times 6150$.)

Fig. 2 Myocardial cell nucleus of a 52-year-old patient with aortic valvular stenosis. Nucleus shows both types of inclusions, globular and tubular, in the nucleoplasm. Globular inclusions are mostly confined to the peripheral parts and are probably always in continuity with the nuclear envelope (arrow). The small sized tubular inclusions are particularly abundant in the heterochromatin-rich areas of the nucleoplasm. $M f=$ Myofilaments. $(\times 6400$.) 


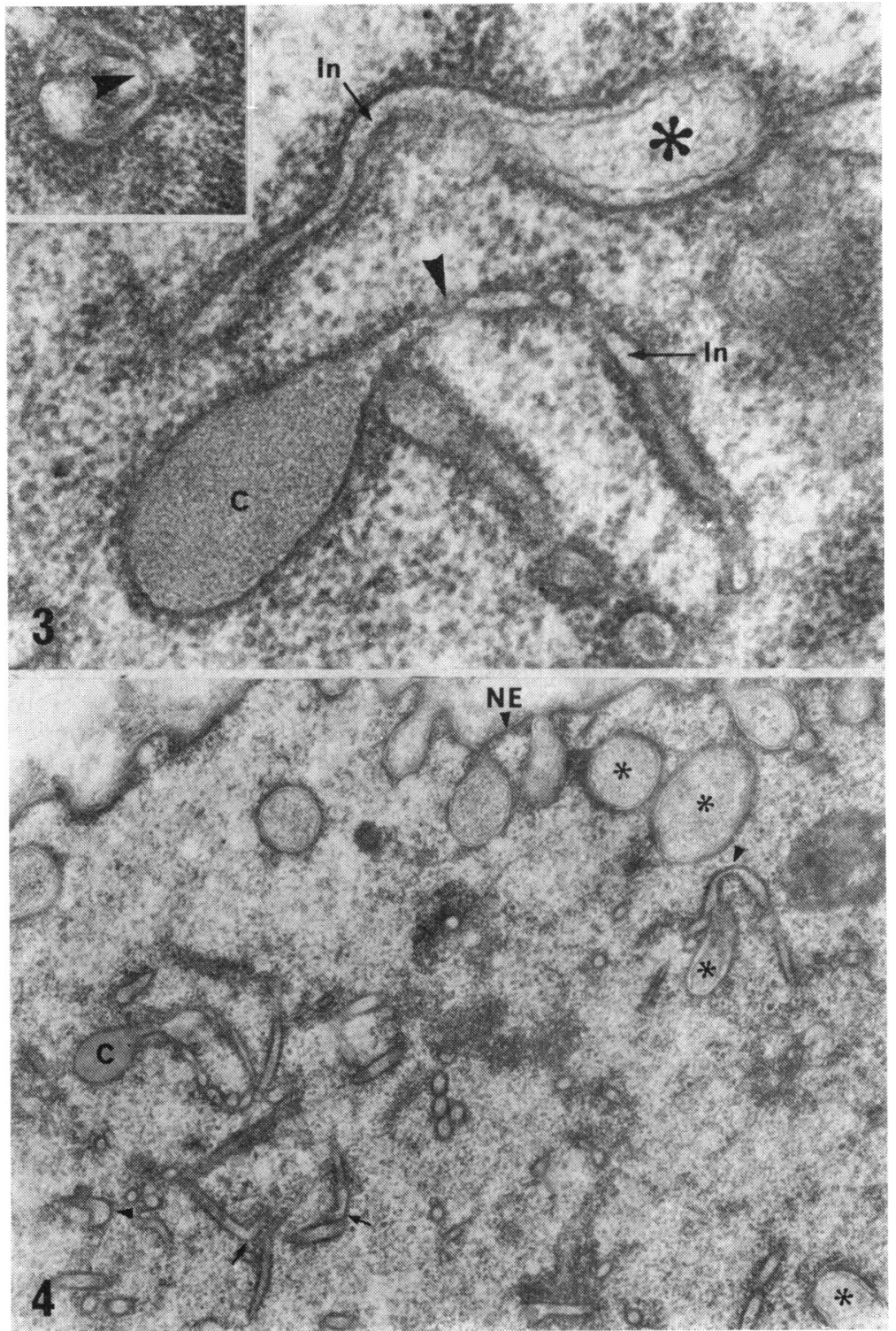

Fig. 3 Part of a myocardial cell nucleus of a 52-year-old patient with aortic valvular stenosis. The micrograph shows intranuclear tubles (In) marginated by heterochromatin. The tubule at the upper left is in continuity with a pseudoinclusion (asterisk), showing that the single-membraned wall is in continuity with the inner nuclear membrane. The tubular dilatation $(C)$ is filled with a granular material. A nuclear pore-like structure is present at arrowhead. Inset shows a segment of a curved tubule with a nuclear pore-like structure at arrowhead. $(\times 54820$.) [Inset : $\times 33770$.]

Fig. 4 Part of a myocardial cell nucleus of a 52-year-old patient with aortic valvular stenosis. The nucleoplasm contains numerous cylindrical and small sized tubules surrounded by heterochromatin. These tubules may run singly, or a few tubules may have their walls closely applied to each other (transversely cut tubules in the centre of the micrograph) Straight or slightly bent segments of the tubules are interrupted by bendings at obtuse or right angles (arrows). Also, curved tubules can be seen (arrowheads). Asterisks=pseudoinclusions, $C=$ tubular cistern; $N E=n u c l e a r$ envelope. $(\times 16500$.) 
ethanol series. The ultrathin sections were gridstained with lead citrate (Reynolds, 1963).

\section{Results}

The nuclear membranes of myocardial cells showed serrated folds caused by the state of contraction (Fig. 1). In addition, the nuclear envelope displayed pseudoinclusions (Fig. 1, 2, 3, and 4) which appeared oval or circular in sections and were not related to cellular contraction. Such structures were mostly confined to the peripheral parts of the nucleus. The membranes of these inclusions were shown to be continuous with those of the nuclear envelope. Yet, probably depending on the plane of sectioning, most of the pseudoinclusions appeared isolated in the nucleoplasm with no visible continuity with the nuclear membranes. The infoldings were lined by both of the membranes of the nuclear envelope and by an outer covering of heterochromatin (Fig. 4). Nuclear pores occurred in these membranes at a seemingly normal frequency. The pseudoinclusions measured about $0.5 \mu$ to $1.5 \mu$ in diameter and contained a granular matrix. Though the latter was occasionally seen to contain ribosomes, other cytoplasmic organelles were not observed in this area. Aberrances of the nuclear envelope, as described above, were noted in 6 of 12 patients and in all three stages examined.

In addition, in 3 of 12 patients, approximately 2 to 3 per cent of the total number of nuclei studied contained cylindrical and small-sized tubules within their nucleoplasm (Fig. 2 to 7). These were lined by a single cell membrane only, shown to be continuous with the inner membrane of the nuclear envelope (Fig. 3). Such tubules mainly occurred in areas of the nucleus rich in heterochromatin. The actual number of intranuclear tubules, as recorded in one single section, varied greatly. While in some nuclei they were only few in number, in others, numerous short and almost straight tubules emanated through the nucleoplasm in different directions (Fig. 4, 7).

Comparisons between different planes of sectioning showed that the intranuclear tubules may run singly, or a few tubules may have their walls so closely applied (Fig. 4) as to appear as bundles in the nucleoplasm. Occasionally, each tubule could be seen to be surrounded by a separate sheath of chromatin of about $160 \AA$ to $200 \AA$ thickness. More frequently, however, the tubules were enclosed in aggregations of heterochromatin of various densities and extensions (Fig. 2, 6, and 7). These aggregations were continuous with the submembraneous heterochromatin of the nucleus
(Fig. 6) and measured about $0.4 \mu$ to $2.0 \mu$ in diameter. The intranuclear tubules ran in straight or slightly bent segments of about $0 \cdot 2 \mu$ to $1.6 \mu$ length, interrupted by bendings at obtuse or right angles (Fig. 4). Now and then, segments of curved tubules were also observed. Tubules not associated with chromatin, and with a clear lumen, were seen in continuity with the former ones (Fig. 5). Such tubules had an irregular course and could be seen to form coiled aggregates (Fig. 7).

The diameter of the tubular lumen measured from $300 \AA$ to $800 \AA$. It was sometimes seen to be continuous with dilated circular sacs filled with a finely granular material (Fig. 3, 4 and 7). A similar material, consisting of somewhat larger granules of moderate electron density, was also found within the tubules (Fig. 5). A rare observation was the presence of nuclear pore-like structures formed by the tubular walls (Fig. 3). In such instances, the apposing membranes of the tubule occluded the lumen for approximately $700 \AA^{\prime}$ length. The marginating chromatin was absent at both sides of the pore-like structure. A close association between the nucleolar material and the intranuclear tubules could not be detected in the present material. In one instance, an aggregation of coiled tubules was seen to be enclosed in electron dense material which appeared similar to the heterochromatin (Fig. 6).

Intranuclear tubules, as described above, were seen in cardiac tissues of 3 patients. In 2 of these, globular infoldings of the nuclear envelope also occurred. These two aberrances of nuclear membranes were often encountered in the same areas of the nucleoplasm. Of the 2 patients mentioned above, one (aged 52 years) with aortic valvular stenosis, had severe hypertrophy of the left ventricular wall. In the other patient (aged 60 years), with aortic valvular regurgitation and mitral valvular stenosis resulting from rheumatic disease, only a light hypertrophy of the left ventricular wall was found. The latter also applied to the third patient (aged 4 years), with congenital ventricular septal defect and pulmonary valvular and infundibular stenosis. In the first and second patient mentioned above, intranuclear tubules were found to be present at all of the three stages examined, i.e. at the start and end of aortic crossclamping and after 20 minutes of reperfusion. In the third patient, biopsy was only taken at the start of cardiopulmonary bypass.

\section{Discussion}

The present study reports two main types of inclusions, globular and tubular, in nuclei of 


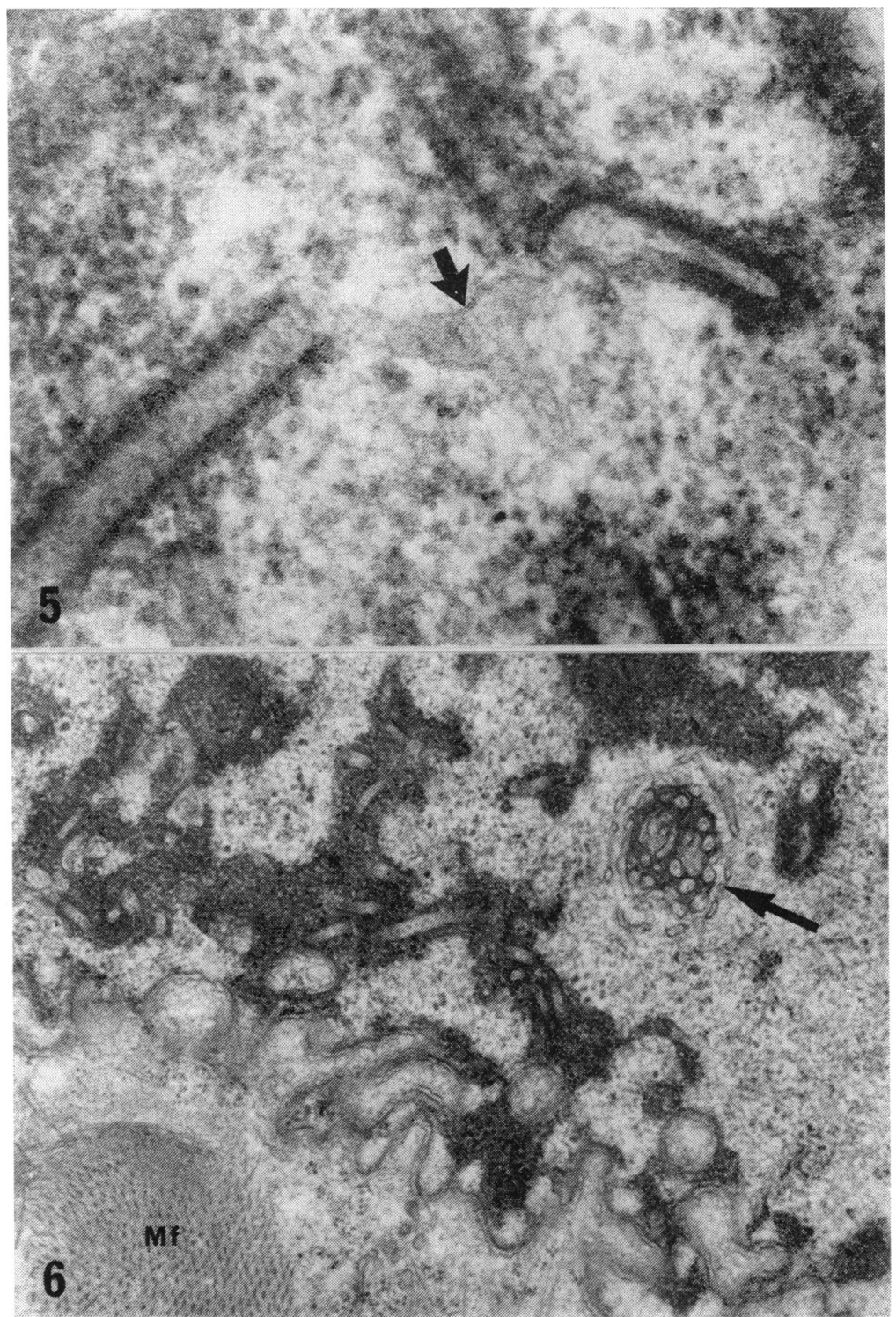

Fig. 5 Part of a myocardial cell nucleus of a 52-year-old patient with aortic valvular stenosis. Intranuclear tubules are presented at a large magnification showing the granular matrix of the tubular lumen and the marginated heterochromatin. A tubule not associated with chromatin (arrow) is continuous with the chromatin-invested one. $(\times 77300$.

Fig. 6 Part of a myocardial cell nucleus of a 60-year-old patient with aortic valvular regurgitation and mitral valvular stenosis caused by rheumatic disease. Intranuclear tubules are 'embedded' in dense condensations of heterochromatin. In addition, segments of a coiled tubule (at arrow) appear closely invested by chromatin-like material. $M f=$ Myofilaments. $(\times 27870$.

human myocardial cells. The globular type of inclusion was lined by a double membrane and superficially located in the nucleus. Such structures were originally described by Bernhard and Granboulan (1963) in cancer cell nuclei and named 'pseudoinclusions'. They have since been found in cell nuclei of a variety of cells (Babai et al., 1969; Sobel et al., 1969; Ferrans et al., 1975).

The pseudoinclusions of the present study are probably always in continuity with the nuclear 


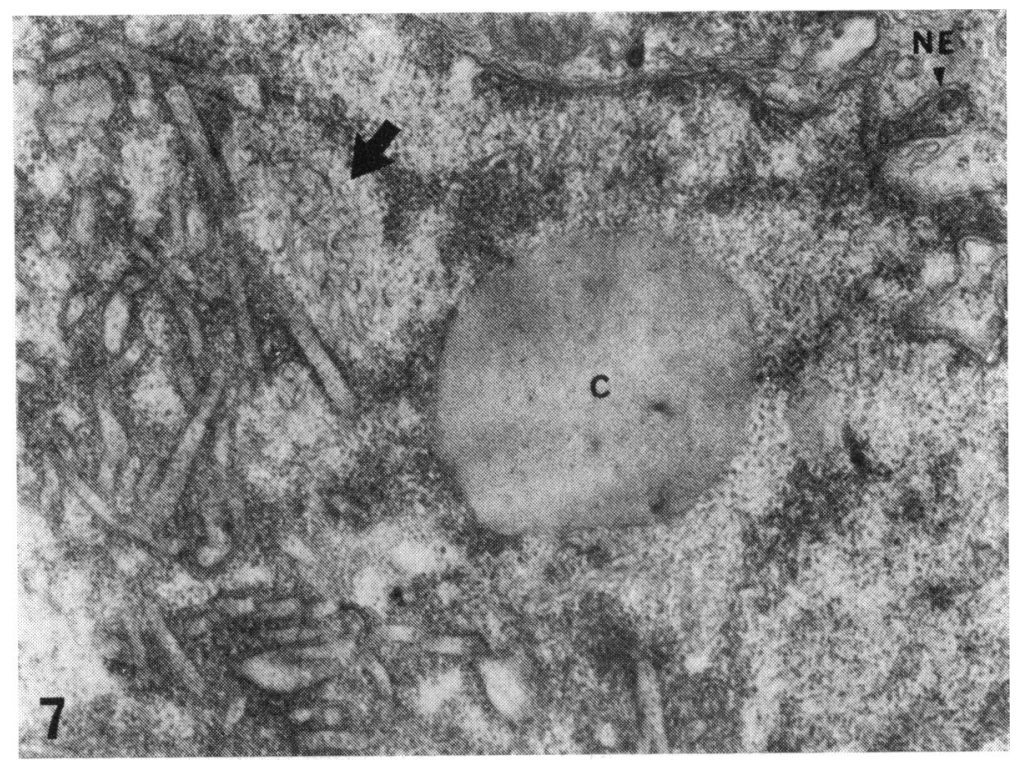

Fig. 7 Part of a myocardial cell nucleus of a 4-year-old patient with congenital ventricular septal defect and pulmonary valvular and infundibular stenosis. In addition to a large number of straight running tubules embedded in heterochromatin, a mass of coiled tubules is present at arrow. $C=$ tubular cistern; NE=nuclear envelope. $(\times 27700$.

envelope even though they often lie seemingly isolated in the submembraneous area. The origin of their lining from the nuclear envelope is evident, though, since it consists of two unit membranes (marginated by heterochromatin), with nuclear pores present at a normal frequency. The functional significance of these nuclear aberrances is still unknown. Previous studies (Babai et al., 1969; Ferrans et al., 1975) have reported that the pseudoinclusions contain cytoplasmic organelles normally present in the perinuclear area. Such cytoplasmic organelles were seen by us only in the larger cytoplasmic invaginations of the nuclear envelope. In our opinion, however, these invaginations are basically different from the globular pseudoinclusions. In the latter, a granular matrix occurred similar to the surrounding chromatin and with no cellular organelles present other than a limited number of ribosomes. Hence, our observations might suggest a functional significance of these nucleic aberrances other than a mere 'trapping' of perinuclear cytoplasm.

Nuclear inclusions which have been previously described show structural similarities to the second type of inclusions reported in the present paper. These are tubular structures lined by a single membrane. In human endometrial cell nuclei (Clyman, 1963; Ancla and DeBrux, 1965; Terzakis, 1965), ringshaped and tubular structures have been shown to be closely connected with the nucleolus, and, also in continuity with the perinuclear space. In the nuclei of an intercranial sarcoma cell, a Golgi-like structure has been reported (Bucciarelli, 1966) in continuity with the inner nuclear membrane. In a recent report, Ferrans et al. (1975) showed the presence of intranuclear tubules derived from the inner nuclear membrane in human cardiac muscle cells. In addition, intranuclear tubules of various configurations have been reported to occur as the result of cardiac viral infections (Middelkamp et al., 1967; Patrizi et al., 1967).

It appears from the present study that intranuclear tubules, similar to those reported above, nearly always are found in areas of the nucleoplasm rich in heterochromatin. Yet, the tubules are not merely channels in the chromatin. The presence of a trilaminar unit membrane encircling the tubular lumen is evident. This is particularly clear in areas where the chromatin invested tubules are continuous with tubules not surrounded by chromatin (Fig. 5).

The often dense packing of tubules at varying sizes, and their close proximity to the heterochromatin seems to be a characteristic feature. In considering their mode of formation, therefore, the possibility cannot be entirely excluded that the tubules arise by the synthesis of membranes 
within the nucleoplasm. Such a view, however, is hardly favoured by two of the present observations, namely the continuity between the tubular membrane and the inner nuclear membrane, and, by the presence of nuclear pores in the tubular wall (Fig. 3). With regard to the latter, is it well known that in rapidly dividing cells, such as malignant cells and öocytes, nuclear pore-like structures do occur in 'annulate lamellae' of the endoplasmic reticulum. These are considered as complicated foldings of the nuclear envelope. To our knowledge, nuclear pore-like structures have not previously been reported in intranuclear tubules derived from the inner nuclear membrane.

The straight or slightly bent intranuclear tubules presented by us, are structurally in accord with those described by Babai et al. (1969) in Novikoff hepatoma cells and by Ferrans et al. (1975) as 'tubules of the first type' in human cardiac muscle cells. Yet, a significant observation of the present study, not reported in earlier works, is the presence of pronounced tubular bendings at the points of termination of the straight running segments.

In addition, the coiled tubules occasionally observed by us in the nucleoplasm, show similarities to those described by Ferrans et al. (1975) as, 'tubules of the second type'. But, in our study such tubules can also be seen to be surrounded by electron dense and chromatin-like material (Fig. 6). Still another significant observation was that pseudoinclusions and intranuclear inclusions were often encountered within the same areas of the nucleoplasm. This might suggest either a common course of formation, or, a functional relation between the two types of membrane aberrances.

Nuclear abnormalities, as described above, were never seen by us in normal mammalian myocardium. They could hardly be caused by cardiac viral infection since viral particles were never seen in the tissues. Both types of inclusions were found at all of the stages examined in the present study. Accordingly, they could not be a reaction to the ischaemia of the heart wall. The alternative, therefore, should be considered that the nuclear aberrances reported might represent a specific response to the process of cardiac hypertrophy (Ferrans et al., 1975).

\section{References}

Ancla, M., and DeBrux, J. (1965). Occurrence of intranuclear tubular structures in the human endometrium during the secretory phase, and of annulate lamellae in hyperestrogenic states. Obstetrics and Gynecology, 26, 23-33.

Babai, F., Tremblay, G., and Dumont, A. (1969). Intranuclear and intranucleolar tubular structures in Novikoff hepatoma cells. Fournal of Ultrastructure Research, 28, 125-130.

Bernhard, W., and Granboulan, N. (1963). The fine structure of the cancer cell nucleus. Experimental Cell Research, 9, Suppl., 19-53.

Bucciarelli, E. (1966). Intranuclear cisternae resembling structures of the Golgi complex. Fournal of Cell Biology, 30, 664-665.

Clyman, M. J. (1963). A new structure observed in the nucleolus of the human endometrial epithelial cell. American fournal of Obstetrics and Gynecology, 86, 430-432.

Ferrans, V. J., Jones, M., Maron, B. J., and Roberts, W. C. (1975). The nuclear membranes in hypertrophied human cardiac muscle cells. American fournal of Pathology, 78, 427-460.

Jensen, H., Engedal, H., and Sætersdal, T. S. (1976). Ultrastructure of mitochondria-containing nuclei in human myocardial cells. Virchows Archiv Abteilung $B$. Zellpathologie, 21, 1-12.

Karnovsky, M. J. (1965). A formaldehyde-gluteraldehyde fixative of high osmolality for use in electron microscopy. fournal of Cell Biology, 27, 137A.

Middelkamp, J. N., Patrizi, G., and Reed, C. A. (1967). Light and electron microscopic studies on the guinea-pig cytomegalovirus. Fournal of Ultrastructure Research, 18, 85-101.

Patrizi, G., Middelkamp, J. N., and Reed, C. A. (1967). Reduplication of nuclear membranes in tissue-culture cells infected with guinea-pig cytomegalovirus. American fournal of Pathology, 50, 779-790.

Rake, M. O., Ansell, I. D., Murray-Lyon, I. M., and Williams, R. (1969). Improved liver-biopsy needle. Lancet, 2, 1283.

Reynolds, E. S. (1963). The use of lead citrate at high pH as an electron opaque stain in electron microscopy. Fournal of Cell Biology, 17, 208-212.

Sobel, H. J., Schwarz, R., and Marquet, E. (1969). Nonviral nuclear inclusions. I. Cytoplasmic invaginations. Archives of Pathology, 87, 179-192.

Sætersdal, T. S., Myklebust, R., Skagseth, E., and Engedal, H. (1976). Ultrastructural studies on the growth of filaments and sarcomeres in mechanically overloaded human hearts. Virchows Archiv Abteilung B. Zellpathologie, 21, 91-112.

Terzakis, J. A. (1965). The nucleolar channel system of human endometrium. Fournal of Cell Biology, 27, 293-304.

Requests for reprints to Dr. Hogne Engedal, Division of Cardiac Surgery, Bergen University Hospital, Haukelandsveien 17, N-5000 Bergen, Norway. 